\title{
Optimal correction of distinct CFTR folding mutants in rectal cystic fibrosis organoids
}

\author{
Johanna F. Dekkers' ${ }^{1,2}$, Ricardo A. Gogorza Gondra ${ }^{1,2}$, Evelien Kruisselbrink ${ }^{1,2}$, \\ Annelotte M. Vonk ${ }^{1,2}$, Hettie M. Janssens ${ }^{3}$, Karin M. de Winter-de Groot ${ }^{1}$, \\ Cornelis K. van der Ent ${ }^{1}$ and Jeffrey M. Beekman ${ }^{1,2}$ \\ Affiliations: 'Dept of Pediatric Pulmonology, Wilhelmina Children's Hospital, University Medical Centre, \\ Utrecht, The Netherlands. 'Laboratory of Translational Immunology, Wilhelmina Children's Hospital, \\ University Medical Centre, Utrecht, The Netherlands. ${ }^{3}$ Dept of Pediatric Pulmonology, Sophia Children's \\ Hospital/Erasmus MC-University Medical Center, Rotterdam, The Netherlands.
}

Correspondence: Jeffrey M. Beekman, Dept of Pediatric Pulmonology, Division of Pediatrics, University Medical Centre Utrecht, Lundlaan 6, HP01.069.0, 3584 EA Utrecht, The Netherlands.

E-mail: J.Beekmandumcutrecht.nl

ABSTRACT Small-molecule therapies that restore defects in cystic fibrosis transmembrane conductance regulator (CFTR) gating (potentiators) or trafficking (correctors) are being developed for cystic fibrosis (CF) in a mutation-specific fashion. Options for pharmacological correction of CFTR-p.Phe508del (F508del) are being extensively studied but correction of other trafficking mutants that may also benefit from corrector treatment remains largely unknown.

We studied correction of the folding mutants CFTR-p.Phe508del, -p.Ala455Glu (A455E) and -p.Asn1303Lys (N1303K) by VX-809 and 18 other correctors (C1-C18) using a functional CFTR assay in human intestinal CF organoids.

Function of both CFTR-p.Phe508del and -p.Ala455Glu was enhanced by a variety of correctors but no residual or corrector-induced activity was associated with CFTR-p.Asn1303Lys. Importantly, VX-809induced correction was most dominant for CFTR-p.Phe508del, while correction of CFTR-p.Ala455Glu was highest by a subgroup of compounds called bithiazoles (C4, C13, C14 and C17) and C5.

These data support the development of mutation-specific correctors for optimal treatment of different CFTR trafficking mutants, and identify $\mathrm{C} 5$ and bithiazoles as the most promising compounds for correction of CFTR-p.Ala455Glu.

@ERSpublications

CFTR corrector efficacy selectively depends on the type of folding and trafficking mutation http://ow.ly/ZrrzB

Received: July 222015 | Accepted after revision: March 072016 | First published online: April 212016

Support statement: This work was supported by grants of the Dutch Cystic Fibrosis Foundation (NCFS) as part of the HIT-CF programme, the Wilhelmina Children's Hospital (WKZ) Foundation and the Dutch Health Organization ZonMw. Funding information for this article has been deposited with FundRef.

Conflict of interest: Disclosures can be found alongside the online version of this article at erj.ersjournals.com 


\section{Introduction}

Loss-of-function mutations in the cystic fibrosis transmembrane conductance regulator (CFTR) gene cause the autosomal-recessive disorder cystic fibrosis (CF). Whereas therapies for CF have historically targeted disease symptoms, novel therapies are being developed that target mutation-specific defects of the CFTR protein, such as repair of 1) CFTR apical trafficking by correctors (e.g. VX-809) [1] and 2) CFTR gating by potentiators (e.g. VX-770) [2]

Most correctors have been identified from screens using CFTR-p.Phe508del (F508del), the most common trafficking mutant expressed by $\sim 90 \%$ of all CF subjects [3]. Recently, the corrector VX-809 (lumacaftor) has been clinically approved for p.Phe508del homozygous subjects in combination with VX-770 (ivacaftor/ Kalydeco) [4, 5], demonstrating for the first time that mutation-specific corrector therapy is feasible. Multiple correctors that have been identified in previous screens for p.Phe508del, but failed to reach the clinical phase, are distributed by Cystic Fibrosis Foundation Therapeutics (CFFT) (Bedford, MA, USA) for experimental purpose (termed C1-C18) [6-12]. While corrector-based treatment options are being extensively studied for CFTR-p.Phe508del, the efficacy of known correctors for other trafficking mutants remains largely unknown and has thus far not been studied in primary epithelial CF cells.

We have recently developed an assay in human primary intestinal organoids [13-15] to study residual and drug-corrected function of mutant CFTR [14, 16]. Forskolin-induced swelling (FIS) of organoids quantitates CFTR function in a subject-specific manner, and was used to characterise correction of the trafficking mutants CFTR-p.Phe508del, -p.Ala455Glu (A455E) or -p.Asn1303Lys (N1303K) by VX-809 and $\mathrm{C} 1-\mathrm{C} 18$.

\section{Methods}

Compounds

VX-809 (Selleck Chemicals LLC, Houston, TX, USA) and C1-C18 (CFFT) were ordered as dry powder. Dimethylsulfoxide stock solutions ( $10 \mathrm{mM}$ for $\mathrm{C} 2$ and $20 \mathrm{mM}$ for all other compounds) were prepared simultaneously for all compounds, stored at $-80^{\circ} \mathrm{C}$ and used within 6 months after storage. Stock solutions were refrozen $\left(-20^{\circ} \mathrm{C}\right)$ a maximum of two times or stored at $-20^{\circ} \mathrm{C}$, maximally for 1 month.

\section{Human material}

Approval for this study was obtained by the Ethics Committee of the University Medical Centre Utrecht (Utrecht, the Netherlands) and the Erasmus Medical Centre Rotterdam (Rotterdam, the Netherlands). Informed consent was obtained from all subjects participating in the study. Organoids from healthy controls and CF subjects (CFTR genotypes indicated in figure 1) were generated from four rectal suction biopsies after intestinal current measurements obtained 1) during standard CF care, 2) for diagnostic purposes or 3) during voluntary participation in studies.

\section{Crypt isolation and organoid culture from rectal suction biopsies}

Methods were slightly adapted from protocols described previously $[14,16]$. In short, crypts were isolated and seeded in 50\% Matrigel (growth factor reduced, phenol-free; BD Biosciences, Breda, the Netherlands) in 24-well plates $(\sim 10-30$ crypts in three $10-\mu \mathrm{L}$ matrigel droplets per well). Growth medium [16] was further supplemented with Primocin (diluted 1 in 500; InvivoGen, Toulouse, France). Vancomycin and gentamycin (both from Sigma, Zwijndrecht, the Netherlands) were added during the first week of culture. The medium was refreshed every $2-3$ days and organoids were passaged $\sim 1$ in 5 every 7-10 days.

\section{The FIS assay}

Methods to measure forskolin-induced organoid swelling described previously [16] were slightly adapted. In short, rectal CF organoids (passage 1-30) from a 7-10-day-old culture were seeded in a flat-bottom 96-well culture plate (Nunc, Rochester, NY, USA) in $5 \mu \mathrm{L} 50 \%$ Matrigel commonly containing 20-80 organoids immersed in $100 \mu \mathrm{L}$ complete culture medium, with or without $3 \mu \mathrm{M}$ VX-809 or $\mathrm{C} 1-\mathrm{C} 18$ at concentrations as indicated in figure 2. 1 day after seeding, organoids were incubated for 30 min with $3 \mu \mathrm{M}$ calcein green (Invitrogen, Carlsbad, CA, USA) in complete culture medium. After calcein green staining, forskolin was added at concentrations as indicated in the figures and organoids were directly analysed by confocal live cell microscopy (LSM710 (Zeiss, Oberkochen, Germany), 5× objective) for $60 \mathrm{~min}$ (p.Phe508del/p.Phe508del and p.Phe508del/p.Ala455Glu) or $120 \mathrm{~min}$ (p.Phe508del/class I and p.Phe508del/p.Asn1303Lys). Two (figures 1 and 2) or three (figure 3) wells were used per condition. Per individual, each experiment was repeated at three to five different time-points.

\section{Quantification of FIS}

Forskolin-stimulated organoid swelling was automatically quantified using Volocity imaging software (Improvision, Coventry, UK). The total organoid area ( $x y$-plane) increase relative to $\mathrm{t}=0 \mathrm{~min}$ of forskolin 
a)
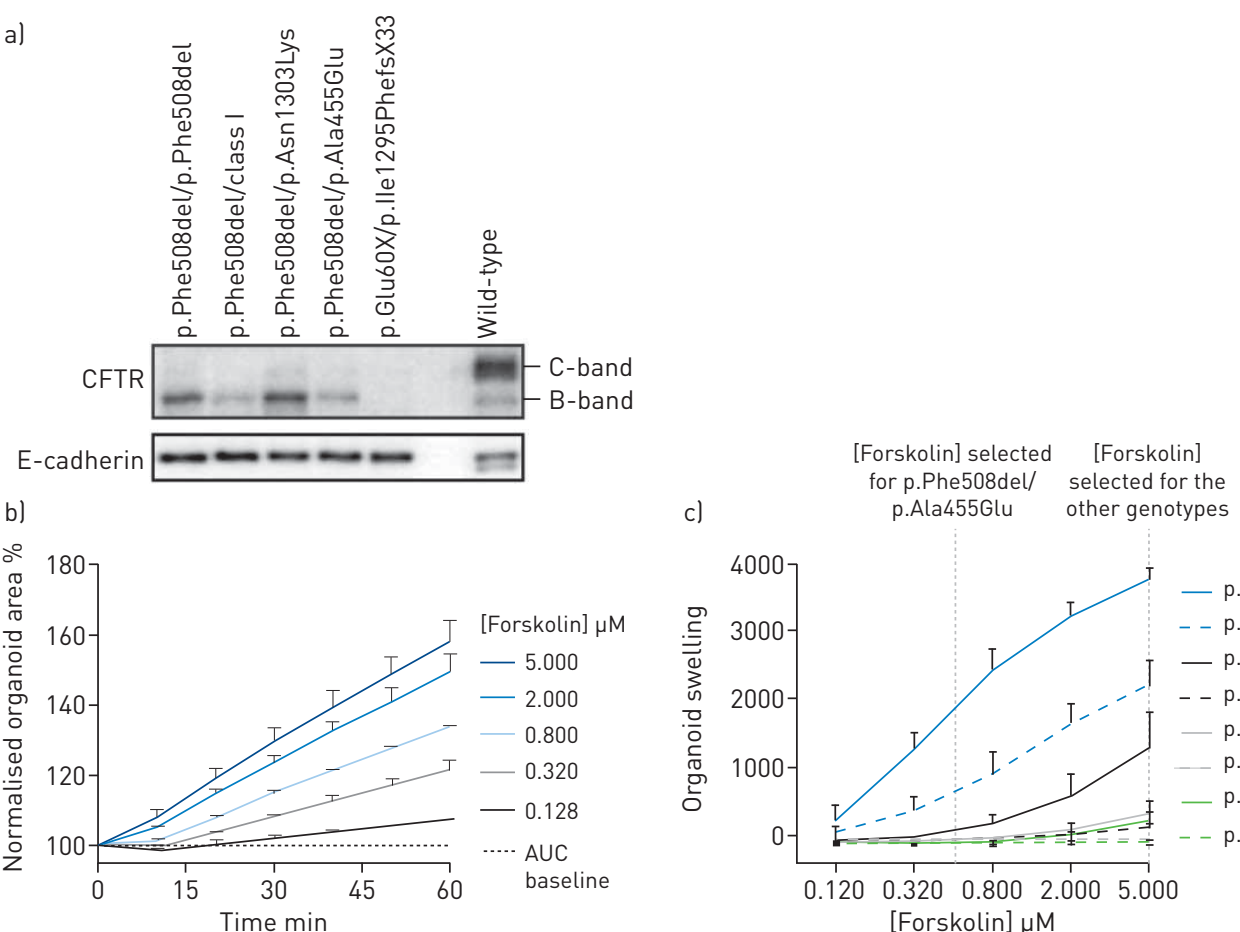

d) p.Phe508del/p.Phe508del
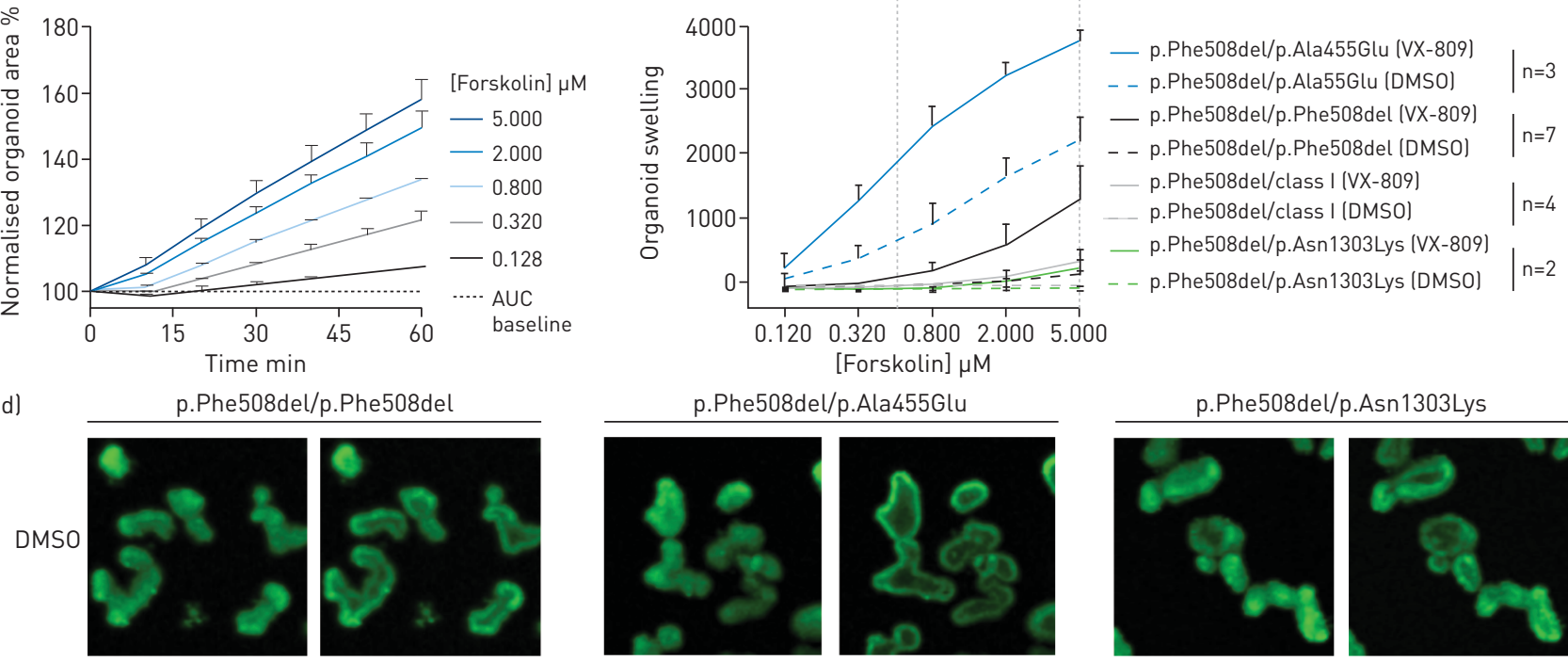

p.Phe508del/p.Ala455Glu
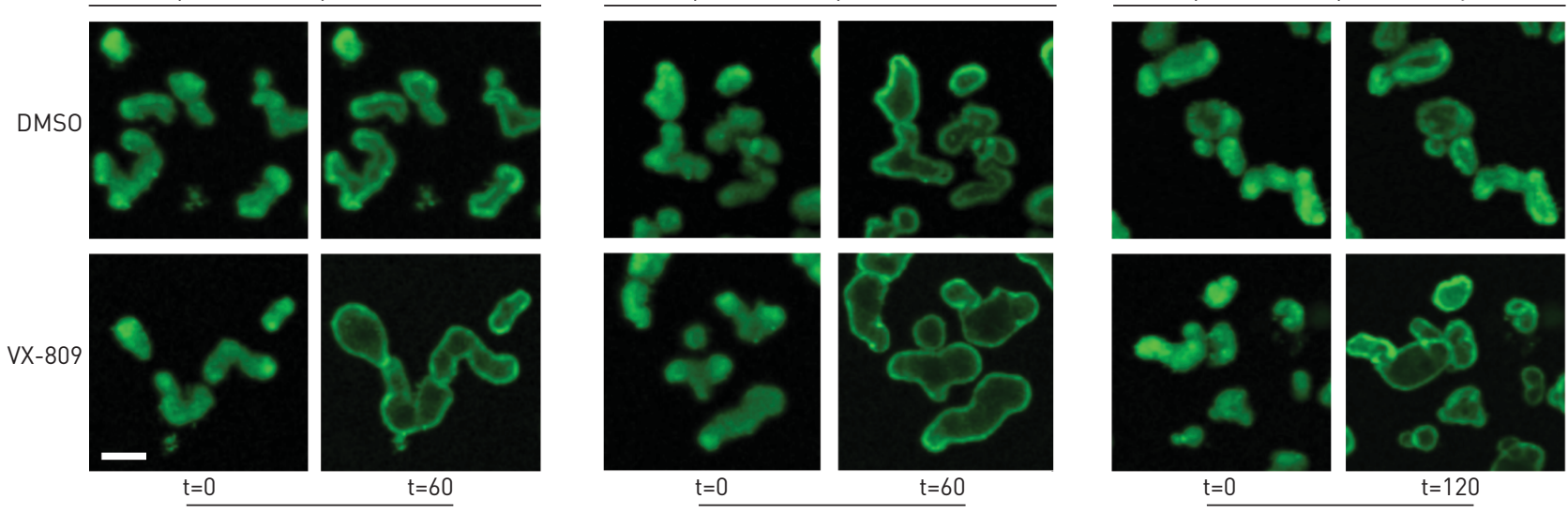

$t=0$

$t=60$

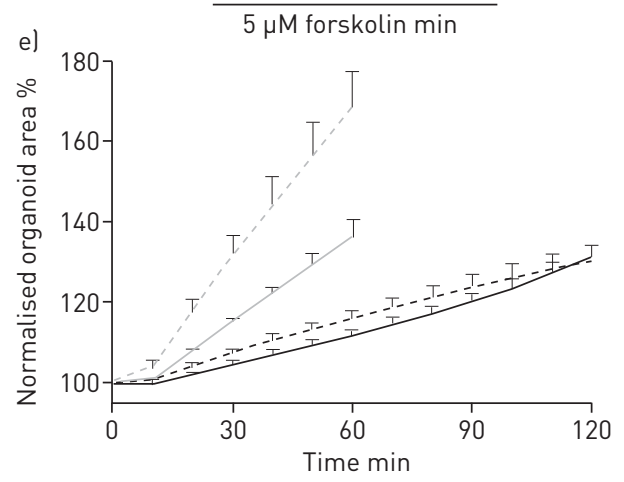

$0.5 \mu \mathrm{M}$ forskolin min

$t=0$

$t=120$

$5 \mu \mathrm{M}$ forskolin min

[Forskolin] $\mu \mathrm{M}$

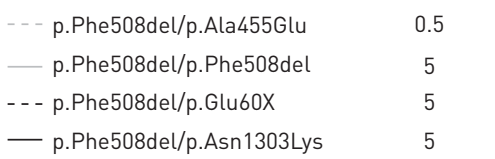

FIGURE 1 Definition of optimal assay conditions for intestinal organoids expressing distinct cystic fibrosis transmembrane conductance regulator (CFTR) mutations. a) Western blot detection of immature (B-band) and mature (C-band) CFTR in organoids with various CFTR genotypes. b) Time tracing of the forskolin-induced surface area increase relative to $\mathrm{t}=0 \mathrm{~min}$ (normalised area) of $\mathrm{p}$.Phe508del/p.Ala455Glu organoids at different forskolin concentrations averaged from two independent wells. c) Forskolin-induced swelling of organoids expressing various CFTR mutations with or without $24 \mathrm{~h}$ pre-treatment with VX-809 $(3 \mu \mathrm{M})$, expressed as the absolute area under the curve (AUC) calculated from time tracings shown in (b) (baseline $100 \%$, $t=60$ min). The class I mutations include p.Glu60X (E60X), p.Gly542X (G542X), p.Arg1162X (R1162X) and p.Ser18ArgfsX16 (DELE2,3). n-values represented the number of CF subjects; each subject was measured at two to five independent culture time-points in duplicate. d) Representative confocal images of calcein green-labelled CF organoids with or without $24 \mathrm{~h}$ pre-treatment with VX-809 at the indicated time-points of forskolin stimulation. Scale bar $=100 \mu \mathrm{m}$. e) Example time tracing of VX-809 $(3 \mu \mathrm{M})$-corrected organoids stimulated with 0.5 or $5 \mu \mathrm{M}$ forskolin and analysed for 60 or 120 min, depending on the CFTR genotype. Data were averaged from two independent wells. All data are presented as mean \pm SD. DMSO: dimethylsulfoxide. 

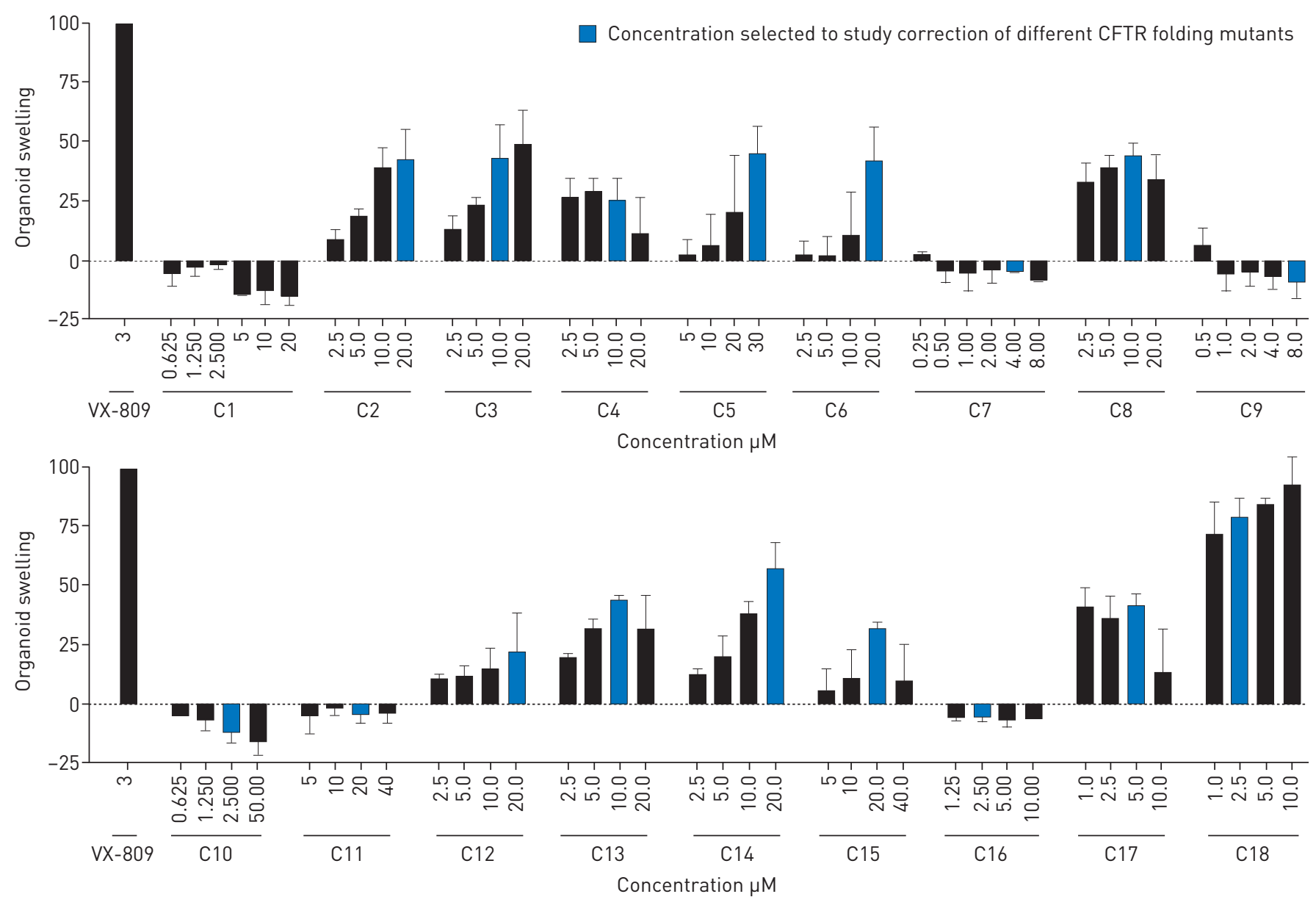

FIGURE 2 Definition of optimal corrector concentrations for cystic fibrosis transmembrane conductance regulator (CFTR)-p.Phe508del. Forskolin-induced swelling (FIS) of p.Phe508del homozygous organoids pre-treated for $24 \mathrm{~h}$ with VX-809 (3 $\mu \mathrm{M})$ or titrations of C1-C18, expressed as the area under the curve calculated from time tracings shown in figure $1 \mathrm{~b}$ (baseline $100 \%, t=60 \mathrm{~min}$ ). Responses were corrected for the FIS of dimethylsulfoxide-treated organoids and normalised to the VX-809 condition. Data were averaged from three independent experiments measured in duplicate, and are presented as mean \pm SD.

treatment was calculated and averaged from two individual wells per condition. In some cases, cell debris and unviable structures were manually excluded based on criteria described in detail in a standard operating procedure. The area under the curve (AUC) ( $\mathrm{t}=60$ or $120 \mathrm{~min}$, baseline $100 \%$ ) was calculated using Prism (GraphPad, La Jolla, CA, USA).

\section{Western blot analysis}

Organoids that were untreated or treated with VX-809 or C17 were lysed in Laemmli buffer supplemented with complete protease inhibitor tablets (Roche, Almere, the Netherlands). Lysates were analysed by SDS-PAGE and electrophoretically transferred to a polyvinylidene difluoride membrane (Millipore, Billerica, MA, USA). The membrane was blocked with $5 \%$ milk protein in Tris-buffered saline-Tween (0.3\% Tween, $10 \mathrm{mM}$ Tris $(\mathrm{pH} 8)$ and $150 \mathrm{mM}$ sodium chloride in water) and probed for $3 \mathrm{~h}$ at room temperature with mouse monoclonal E-cadherin-specific (1 in 10000 dilution; BD Biosciences) or CFTR-specific antibodies (Cystic Fibrosis Folding Consortium 450, 570 and 596; 1 in 3000 dilution;), followed by incubation with horseradish peroxidase-conjugated secondary antibodies and enhanced chemiluminescence development.

\section{Results}

Defining optimal assay conditions for intestinal organoids expressing distinct CFTR mutations

To define optimal assay conditions for organoids expressing different CFTR mutations, swelling of organoids derived from subjects homozygous for p.Phe508del, or compound heterozygous for p.Phe508del and p.Ala455Glu, p.Asn1303Lys or a class I (resulting in no functional CFTR protein) mutation was assessed at five different forskolin concentrations with or without VX-809 (figure 1). We first confirmed 

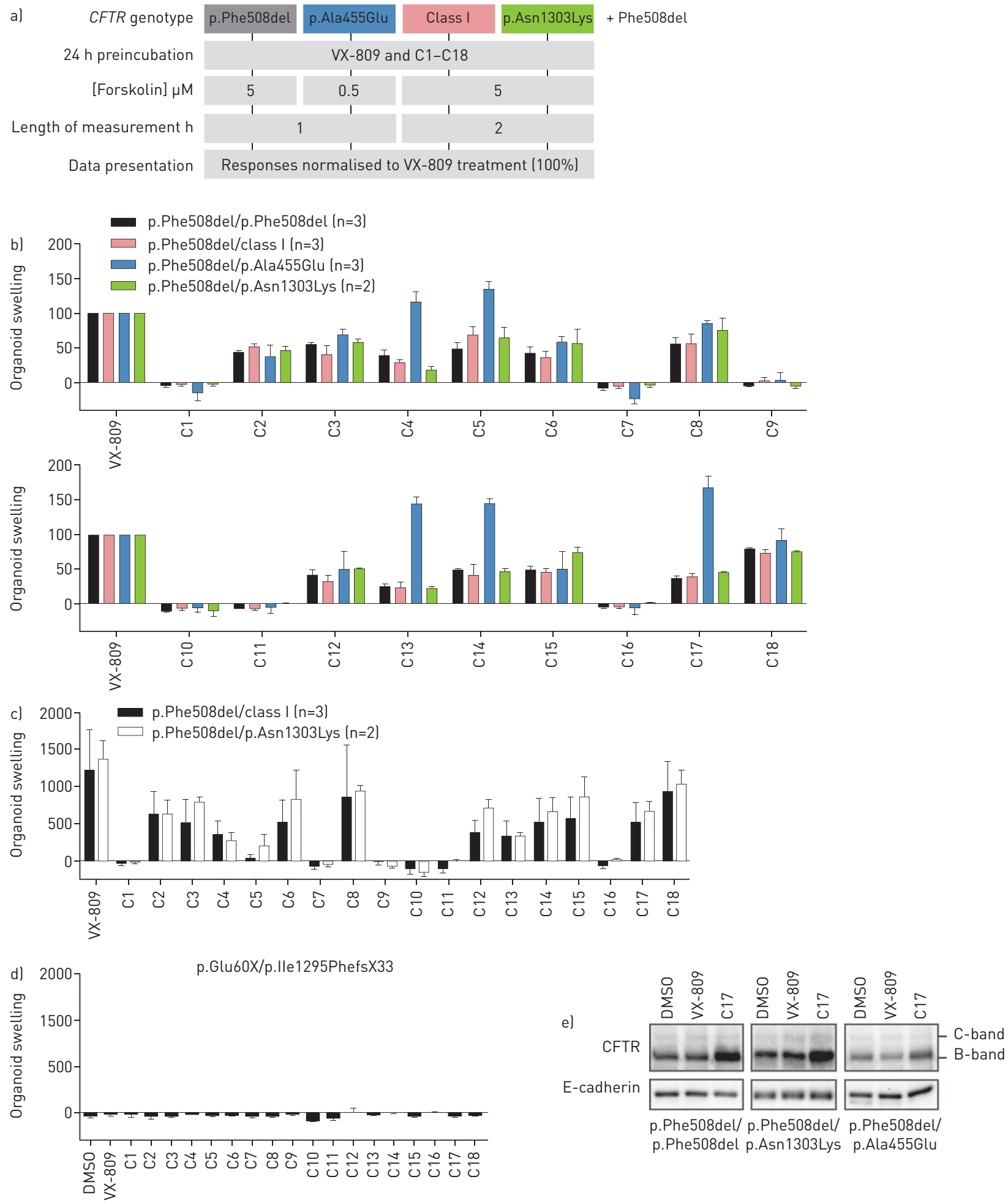

FIGURE 3 Correction of cystic fibrosis transmembrane conductance regulator (CFTR)-p.Phe508del, -p.Ala455Glu or -p.Asn1303Lys by VX-809 and C1-C18. a) Overview of CFTR genotype-specific assay conditions. b, c) Forskolin-induced swelling (FIS) of organoids expressing various CFTR mutations pre-treated for $24 \mathrm{~h}$ with VX-809 $(3 \mu \mathrm{M})$ or $\mathrm{C} 1-\mathrm{C} 18$ using concentrations defined in figure 2. Responses were corrected for the FIS of dimethylsulfoxide (DMSO)-treated organoids and expressed as the normalised (b) or absolute (c) area under the curve calculated from the time tracings shown in figure $1 \mathrm{~b}$ (baseline $100 \%, \mathrm{t}=60$ or $120 \mathrm{~min}$ ). d) FIS of corrector-stimulated organoids expressing two class I mutations. b-d) The nonsense mutations include p.Glu60X (E60X), p.Gly542X (G542X) and p.Arg1162X (R1162X). n-values represent the number of CF subjects; each subject was measured at two independent culture time-points in triplicate. Date are presented as mean \pm SD. e) Western blot detection of immature (B-band) and mature (C-band) CFTR in organoids expressing various CFTR mutations upon treatment with DMSO, VX-809 (3 $\mu$ M) or C17 ( $5 \mu$ ). Results are representative for three (C17) or four (DMSO and VX-809) independent experiments using organoids derived from two different donors per genotype (similar to donors used in b, c). 
folding and trafficking defects in CFTR-p.Phe508del, -p.Ala455Glu and -p.Asn1303Lys by Western blot analysis, which indicated highly reduced levels of complex-glycosylated CFTR (C-band) compared to wild-type CFTR organoids (figure 1a). No CFTR expression was detected in organoids expressing two class I mutations, confirming specificity for CFTR (figure 1a). As described previously [16], organoid swelling was presented as absolute AUC calculated from 60-min time tracings of the surface area increase of calcein green-labelled organoids relative to $\mathrm{t}=0 \mathrm{~min}$ (figure $1 \mathrm{~b}$ and $\mathrm{c}$ ). The basal and VX-809-induced swelling was forskolin dose-dependent and highest for p.Phe508del/p.Ala455Glu organoids (figure 1c). FIS of p.Phe508del/p.Asn1303Lys and p.Phe508del/class I organoids was similar, suggesting that only limited to no function was associated with p.Asn1303Lys. Based on figure $1 \mathrm{c}$, we selected $0.5 \mu \mathrm{M}$ forskolin for p.Phe508del/p.Ala455Glu and $5 \mu \mathrm{M}$ forskolin for the other genotypes to study corrector effects of C1-C18. Of note, FIS of p.Phe508del/p.Ala455Glu organoids at $0.5 \mu \mathrm{M}$ forskolin is mainly conferred by the CFTR-p.Ala455Glu allele, as the responses of p.Phe508del/class I organoids were near background level at this forskolin dose (figure 1c). FIS of organoids compound heterozygous for p.Phe508del and p.Asn1303Lys or a class I mutation was analysed for 120 instead of $60 \mathrm{~min}$ to increase assay sensitivity (figure $1 \mathrm{~d}$ and e). To conclude, the assay conditions defined here displayed optimal conditions to assess corrector-repaired FIS of organoids with distinct CFTR folding mutations.

\section{Defining optimal corrector concentrations using CFTR-p.Phe508del homozygous organoids}

We next measured FIS of p.Phe508del/p.Phe508del organoids that were treated with VX-809 or different concentrations of C1-C18 to identify optimal compound dosages (figure 2). The tested dosages for C1-C18 were based on compound potency data described in the CFFT compound order from or in the literature. Compared to FIS of uncorrected organoids, we observed a dose-dependent increase in FIS for most compounds but a similar or reduced FIS for C1, C7, C9, C10, C11 and C16, indicating lack of functional CFTR-p.Phe508del repair by these compounds (figure 2). Reduced FIS may point to compound toxicity. One concentration per compound was selected for further analysis based on figure 2 for the compounds associated with correction or on the literature for the nonfunctional correctors (figure 2). In conclusion, we identified functional and nonfunctional CFTR-p.Phe508del correctors, and selected optimal C1-C18 concentrations for efficacy testing using organoids with distinct trafficking mutants.

\section{Correction of CFTR-p.Phe508del, -p.Ala455Glu and -p.Asn1303Lys by VX-809 and C1-C18}

To study mutation-specific CFTR repair by correctors, FIS of organoids derived from donors homozygous for p.Phe508del or compound heterozygous for p.Phe508del and a nonsense mutation, p.Ala455Glu or p.Asn1303Lys was assessed using assay conditions defined from figures 1 and 2 (figure $3 \mathrm{a}$ ). The data were normalised to VX-809 to correct for CFTR mutation-dependent assay differences (figure $3 \mathrm{~b}$ ). In this way, compounds that selectively act on a specific mutation can be identified. In line with figure 2 , all compounds, except for C1, C7, C9, C10, C11 and C16, corrected FIS of all cultures. The relative correction patterns of p.Phe508del/p.Phe508del, p.Phe508del/class I and p.Phe508del/p.Asn1303Lys organoids were comparable (figure $3 \mathrm{~b}$ ), as well as the absolute correction patterns of p.Phe508del/class I and p.Phe508del/ p.Asn1303Lys organoids (figure 3c), indicating that none of the compounds functionally corrected CFTR-p.Asn1303Lys. Interestingly, FIS was greatly increased by C4, C5, C13, C14 and C17 specifically for p.Phe508del/p.Ala455Glu organoids, reaching $120-170 \%$ of the response to VX-809 (100\%), while VX-809 was the most dominant corrector for the other genotypes (figure 3b). Of these p.Ala455Glu-specific compounds, C5 contains a phenylquinoline group, and C4, C13, C14 and C17 share a bithiazole chemical structure (www.ebi.ac.uk/chembl/). Swelling was absent in organoids expressing two class I mutations, indicating corrector-induced FIS is fully CFTR-dependent (figure 3d). Western blot studies indicated that C17, but not VX-809, increased immature CFTR (B-band) levels for all mutants (figure 3e). With or without corrector treatment, expression of maturated CFTR-p.Phe508del or CFTR-p.Ala455Glu (C-band) was near background levels and difficult to evaluate. However, data suggested that VX-809 and C17 increased CFTR-p.Asn1303Lys C-band (figure 3e), which was in contrast with the lack of functional CFTR-p.Asn1303Lys repair by these correctors (figure $3 b$ and c). Together, these data indicate that optimal correction of distinct trafficking mutations requires different chemical correctors.

\section{Discussion}

We assessed, for the first time, correction of multiple CFTR trafficking mutants in primary cells by assessment of the FIS assay in rectal organoids using 19 chemical correctors [16, 17]. As residual function for the different trafficking mutants was different (figure $1 \mathrm{c}$ and e), assay conditions were adapted to enable optimal detection of correction of the various mutants (figure 3a). Organoids with p.Ala455Glu have considerable residual function and the forskolin dose was reduced to enable optimal detection of corrector efficacy. In contrast, for p.Phe508del/class I and p.Phe508del/p.Asn1303Lys, the FIS measurement was prolonged from 60 to $120 \mathrm{~min}$ to increase assay sensitivity. Normalisation of the 
corrector-repaired datasets to VX-809 allowed comparison of the four different genotypes and to pinpoint functional correction specifically to CFTR-p.Ala455Glu or -p.Asn1303Lys (figure 3b).

This approach indicated that repair of CFTR-p.Ala455Glu was highest by a subgroup of four correctors sharing a bithiazole group and by C5, containing a phenylquinoline group (figure 3b). Recent data already indicated that corrector-mediated repair of CFTR-p.Ala455Glu function (unpublished obervations) and expression [18] is feasible, but the preference of certain correctors for CFTR-p.Ala455Glu compared to CFTR-p.Phe508del has never been shown. These findings suggest that CFTR-p.Ala455Glu and CFTR-p. Phe508del contain distinct folding defects. Recent observations indicated that the bithiazole C4 acts as a class II corrector, targeting the nucleotide-binding domain (NBD)2-membrane-spanning domain (MSD)1 interface, which repairs CFTR-p.Phe508del expression and function synergistically when combined with class I correctors (VX-809) that target the NBD1-MSD2 interface [19]. The specific folding defects and corrector target domains in CFTR-p.Ala455Glu need to be further defined.

The similarity between the relative (figure 3b) and absolute (figure 3c) correction profiles of p.Phe508del/ p.Asn1303Lys, p.Phe508del/p.Phe508del and p.Phe508del/class I organoids indicate that FIS of p.Phe508del/p.Asn1303Lys cultures most likely depends on CFTR-p.Phe508del, and that function of CFTR-p.Asn1303Lys is not restored by any $\mathrm{C} 1-\mathrm{C} 18$ corrector. These data support other findings demonstrating that CFTR-p.Asn1303Lys could not be functionally repaired by VX-809 or VX-770 in human intestinal organoids (unpublished observations), Fischer rat thyroid cells [20] and human primary lung cells [21]. In line with ectopic CFTR expression studies in cell lines [22], increased B-band and decreased C-band levels were detected for CFTR-p.Asn1303Lys compared to wild-types (figure 1a). In contrast to the lack of functional CFTR-p.Asn1303Lys repair, protein expression levels of this mutant can be partially restored by correctors [21] (figure 3e). The inconsistency between correction of processing and function suggests that either the folding defect in CFTR-p.Asn1303Lys completely blocks its function at the apical membrane, even when trafficking is partly restored by chemical correctors, or that complex-glycosylated CFTR-p.Asn1303Lys (C-band) is aberrantly targeted to cellular compartments other than the apical membrane. Clearly, development of novel treatment strategies is required to repair the specific defect in CFTR-p.Asn1303Lys that blocks its function.

In conclusion, we studied functional CFTR repair by VX-809 and C1-C18 in primary intestinal CF organoids with different trafficking mutants, and observed correction of CFTR-p.Phe508del and -p.Ala455Glu by 13 out of 19 compounds, while none of these compounds restored function of CFTRp.Asn1303Lys. Most importantly, C5 and a subgroup of compounds that share a bithiazole chemical structure achieved highest repair of CFTR-p.Ala455Glu, while correction of CFTR-p.Phe508del was dominated by VX-809. These data indicate, for the first time in primary human CF cells, that the CFTR corrector efficacy selectively depends on the type of folding and trafficking defect, and support the development of mutation-specific corrector strategies that are optimal for distinct CFTR mutants.

\section{Acknowledgements}

We thank S. Heida-Michel, M. Geerdink, M.C.J. Olling-de Kok (all Dept of Pediatric Pulmonology, Wilhelmina Children's Hospital, University Medical Centre, Utrecht, the Netherlands), E.M. Nieuwhof-Stoppelenburg and E.C. van der Wiel (both Dept of Pediatric Pulmonology, Erasmus University Medical Centre/Sophia Children's Hospital, Rotterdam, the Netherlands) for providing intestinal biopsies; R.J. Bridges (Dept of Physiology and Biophysics, Rosalind Franklin University of Medicine and Science, Chicago, IL, USA) and CFFT for providing the C1-C18 compounds; and J. Riordan (Dept of Biochemistry and Biophysics, University of North Carolina, Chapel Hill, NC, USA) and CFFT for providing the CFTR monoclonal antibodies.

\section{References}

1 Van Goor F, Yu H, Burton B, et al. Effect of ivacaftor on CFTR forms with missense mutations associated with defects in protein processing or function. J Cyst Fibros 2014; 13: 29-36.

2 Yu H, Burton B, Huang CJ, et al. Ivacaftor potentiation of multiple CFTR channels with gating mutations. J Cyst Fibros 2012; 11: 237-245.

3 Cheng SH, Gregory RJ, Marshall J, et al. Defective intracellular transport and processing of CFTR is the molecular basis of most cystic fibrosis. Cell 1990; 63: 827-834.

4 Boyle MP, Bell SC, Konstan MW, et al. A CFTR corrector (lumacaftor) and a CFTR potentiator (ivacaftor) for treatment of patients with cystic fibrosis who have a phe508del CFTR mutation: a phase 2 randomised controlled trial. Lancet Respir Med 2014; 2: 527-538.

5 Wainwright CE, et al. Lumacaftor-ivacaftor in patients with cystic fibrosis homozygous for Phe508del CFTR. N Engl J Med 2015; 373: 220-231.

6 Pedemonte N, Lukacs GL, Du K, et al. Small-molecule correctors of defective $\Delta$ F508-CFTR cellular processing identified by high-throughput screening. J Clin Invest 2005; 115: 2564-2571.

7 Van Goor F, Straley KS, Cao D, et al. Rescue of $\triangle$ F508-CFTR trafficking and gating in human cystic fibrosis airway primary cultures by small molecules. Am J Physiol Lung Cell Mol Physiol 2006; 290: L1117-L1130.

8 Loo TW, Bartlett MC, Wang Y, et al. The chemical chaperone CFcor-325 repairs folding defects in the transmembrane domains of CFTR-processing mutants. Biochem J 2006; 395: 537-542. 
Hirth BH, Qiao S, Cuff LM, et al. Discovery of 1,2,3,4-tetrahydroisoquinoline-3-carboxylic acid diamides that increase CFTR mediated chloride transport. Bioorg Med Chem Lett 2005; 15: 2087-2091.

10 Robert R, Carlile GW, Pavel C, et al. Structural analog of sildenafil identified as a novel corrector of the F508del-CFTR trafficking defect. Mol Pharmacol 2008; 73: 478-489.

11 Macia E, Ehrlich M, Massol R, et al. Dynasore, a cell-permeable inhibitor of dynamin. Dev Cell 2006; 10: 839-850.

12 Yoo CL, Yu GJ, Yang B, et al. 4'-Methyl-4,5'-bithiazole-based correctors of defective $\triangle$ F508-CFTR cellular processing. Bioorg Med Chem Lett 2008; 18: 2610-2614.

13 Sato T, van Es JH, Snippert HJ, et al. Paneth cells constitute the niche for Lgr5 stem cells in intestinal crypts. Nature 2011; 469: 415-418.

14 Sato T, Stange DE, Ferrante M, et al. Long-term expansion of epithelial organoids from human colon, adenoma, adenocarcinoma, and Barrett's epithelium. Gastroenterology 2011; 141: 1762-1772.

15 Jung P, et al. Isolation and in vitro expansion of human colonic stem cells. Nat Med 2011; 17: 1225-1227.

16 Dekkers JF, Wiegerinck CL, de Jonge HR, et al. A functional CFTR assay using primary cystic fibrosis intestinal organoids. Nat Med 2013; 19: 939-945.

17 Dekkers JF, van der Ent CK, Beekman JM. Novel opportunities for CFTR-targeting drug development using organoids. Rare Dis 2013; 1: e27112.

18 Cebotaru L, Rapino D, Cebotaru V, et al. Correcting the cystic fibrosis disease mutant, A455E CFTR. PLoS One 2014; 9: e85183.

19 Okiyoneda T, Veit G, Dekkers JF, et al. Mechanism-based corrector combination restores $\triangle$ F508-CFTR folding and function. Nat Chem Biol 2013; 9: 444-454.

20 Van Goor F, Yu H, Burton B, et al. Effect of ivacaftor on CFTR forms with missense mutations associated with defects in protein processing or function. J Cyst Fibros 2014; 13: 29-36.

21 Awatade NT, Uliyakina I, Farinha CM, et al. Measurements of functional responses in human primary lung cells as a basis for personalized therapy for cystic fibrosis. EBioMedicine 2015; 2: 147-153.

22 Rapino D, Sabirzhanova I, Lopes-Pacheco M, et al. Rescue of NBD2 mutants N1303K and S1235R of CFTR by small-molecule correctors and transcomplementation. PLoS One 2015; 10: e0119796. 